Voix et Images

\title{
De « L’art de la défaite " à Prochain Épisode : un récit unique?
}

\section{Anthony Purdy}

Volume 10, numéro 3, printemps 1985

André Major

URI : https://id.erudit.org/iderudit/200518ar

DOI : https://doi.org/10.7202/200518ar

Aller au sommaire du numéro

\section{Éditeur(s)}

Université du Québec à Montréal

\section{ISSN}

0318-9201 (imprimé)

1705-933X (numérique)

Découvrir la revue

\section{Citer cet article}

Purdy, A. (1985). De « L’art de la défaite » à Prochain Épisode : un récit unique?

Voix et Images, 10(3), 113-125. https://doi.org/10.7202/200518ar d'utilisation que vous pouvez consulter en ligne.

https://apropos.erudit.org/fr/usagers/politique-dutilisation/ 


\title{
De «L'art de la défaite» \\ à Prochain Épisode: un récit unique?
}

\author{
par Anthony Purdy, Université de l'Alberta
}

- Parce que l'histoire ne se fait qu'en se racontant, une critique de l'histoire ne peut être exercée qu'en racontant comment l'histoire, en se narrant, se produit.

- Jean-Pierre Faye

L'histoire est d'abord une narration. Telle est cette «évidence toute primitive» que Jean-Pierre Eaye ne laisse pas de nous rappeler dans ses livres; une narration ou un enchevêtrement de récits, car l'histoire, on ne saurait trop le répéter, ne se fait qu'en se racontant 1 . D'ailleurs, si l'on est obligé de considérer l'histoire comme une narration, c'est-à-dire comme le récit d'une action, il s'ensuit que les effets de cette narration - l'action du récit - font, à leur tour, partie intégrante d'une histoire qui ne cesse jamais de se raconter. Car «il existe, dans l'histoire, un effet de production d'action par le récit», «effet de la narration sur l'action qu'elle est en train de narrer ${ }^{2}$ ». Cet effet producteur du récit, Faye l'a baptisé, on s'en souvient, l'effet Mably et en a fourni le paradigme dans ce «récit hunique» où l'abbé raconte la traversée du Rhin et l'invasion par les «barbares» de la Gaule romaine:

Quelques jeunes Huns chassoient sur les bords du Palus

Méotides; une biche qu'ils avoient lancée traversa un marais qu'ils regardoient comme une mer impraticable; et en suivant témérairement leur proie, ils furent étonnés de se trouver dans un nouveau monde. Ces chasseurs, impatients de raconter à leurs familles les merveilles qu'ils avoient vues, retournèrent dans leur habitation; et les récits par lesquels ils piquoient la curiosité de leurs compatriotes, devoient changer la face des nations. Jamais peuple ne fut plus terrible que les Huns ${ }^{3}$.

Ainsi, à côté des changements matériels - des conditions et des modes de production et d'échange - survenus sur la rive droite du Rhin, intervient «l'événement imprévu de ces 'récits' qui vont 'changer la face des nations': de cette production d'action supplémentaire et, pour ainsi dire, discontinue, par l'effet de récit ${ }^{4}$ ).

Ses premières réflexions sur ce récit collectif, Jean-Pierre Faye les a publiées en mars $1966^{5}$. Un an auparavant, dans un climat politique et intellectuel tout autre, Hubert Aquin avait déjà consacré un article à un phénomène semblable. Il s'agit, bien entendu, de «L'art de la défaite», de ces «considérations stylistiques» sur l'échec de la rébellion de 1837-18386. Rappelons brièvement la thèse d'Aquin, qui a vu dans la rébellion des Patriotes une «véritable anthologie d'erreurs sanglantes, de négligences et 
d'actes manqués»: «Tout était prévisible, tout! Et tout a été prévu; rien n'a été laissé au hasard... La rébellion de 1837-1838 est la preuve irréfutable que les Canadiens français sont capables de tout, voire même de fomenter leur propre défaite...7\%). Mais pourquoi la rébellion aurait-elle été conduite et vécue par les Patriotes comme une guerre perdue d'avance? Aquin croit découvrir un premier indice dans le récit fait par l'abbé Groulx de la victoire imprévue de Saint-Denis: les Patriotes, dit Groulx, n'osèrent pas «profiter de leur victoire et donner la chasse aux compagnies de Gore en pleine déroute ${ }^{8}$ ).

Comment expliquer cette «étrange et mystérieuse défectuosité» de la part d'un groupe d'hommes qui venaient de remporter une victoire exaltante? S'agirait-il, comme le suggère Aquin, d'un blanc de mémoire, d'une sorte d'amnésie ou d'aphasie collective? «On se croirait à la représentation d'une tragédie classique, à l'instant où le choeur, instantanément et dans une invraisemblable simultanéité, a un blanc de mémoire: c'est un silence de mort ${ }^{9}$.» Ou bien, en atténuant l'invraisemblance de l'hypothèse sans pour autant abandonner la métaphore théâtrale, ne pourrait-on pas y voir une confusion provoquée par un événement imprévu: «Le choeur, figé de stupeur, ne peut pas enchaîner si l'action dramatique qui vient de se dérouler n'était pas dans le texte; les Patriotes n'ont pas eu un blanc de mémoire à Saint-Denis, mais ils étaient bouleversés par un événement qui n'était pas dans le texte: leur victoirel0!»

En contrastant la «stylistique» de la rébellion de 1837 avec celle de l'invasion de 1838, en s'attachant au «style suicidaire» de l'art militaire des Patriotes, en comparant leur rébellion à «l'entreprise poétique d'un homme

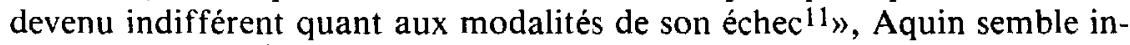
sister sur le caractère littéräire et textuel des événements de Saint-Denis. La défaire des Patriotes de 1837 serait donc avant tout un échec narratif: si leur victoire ne s'est pas produite, c'est qu'il n'ont pas su raconter une histoire invraisemblable, qui n'était pas prévue dans les règles du genre. Ici, comme dans le cas du "récit hunique» de l'abbé Malby, l'histoire est d'abord une narration. Or, les Canadiens français, comme le dira Lord Durham en 1839, ne sont-ils pas précisément ce peuple sans histoire, ou plutôt, selon l'expression d'Aquin, un peuple pris dans «une histoire dont il ne serait jamais l'auteur... une histoire faite par d'autres ${ }^{12}$ ?» Un peuple donc qui serait incapable de se raconter, de s'emparer de la narration de son histoire. Si la victoire des Patriotes de 1837 était inconvenable, c'est que le récit de cette victoire dépassait la compétence narrative des Patriotes.

Ces réflexions sur la défaite des Patriotes ont paru dans la revue Liberté en mars 1965. Elles servent ainsi de préface au roman publié par Aquin en novembre de cette année ${ }^{13}$. On se souviendra que l'action de Prochain Épisode tourne autour des efforts du héros pour tuer son adversaire H. de Heutz, historien des guerres romaines et professeur à l'Université de Bâle 14 , alias Carl von Ryndt, «banquier comme il y en a des milliers en Suisse» (p. 39). Ces tentatives d'assassinat donnent lieu à trois confrontations qui constituent une sorte de combat ritualisé entre le jeune révolution- 
naire et son professeur d'histoire: combat qui se présente comme une analogie des efforts frustrés d'un peuple pour raconter sa propre histoire.

La première confrontation a lieu «quelque part entre Genève et Lausanne» au château d'Echandens. Le héros du récit d'espionnage, qui s'est laissé assommer par ses ennemis, sort de son "sommeil comateux» pour se trouver désarmé et en présence de son adversaire, qu'il ne connaît pas encore sinon par «son invraisemblance et son indétermination» (p. 52 ). Ayant établi qu'il a affaire à un «interlocuteur valable» avec qui un «dialogue positif» serait possible, il cherche les paroles qui lui permettraient de se tirer d'une situation difficile: «Il me fallait élaborer une riposte-éclair et puisque je n'avais plus d'arme à dégainer, vider mon chargeur dialectique sur cet inconmu dressé entre le jour et moìn. (p. 58). Sa seule arme est donc sá compétence narrative, sa capacité d'inventer et de raconter une histoire, fait qui est souligné par le retour immédiat à la situation narrative et à l'impuissance du narrateur «à souffler à son double les quelques phrases d'occasion qui le sortiraient du pétrin" (p. 58). Narrateur et personnage se troúvent comme paralysés, plongés dans une "catatonie nationale» devant cet inconnu «mandaté pour (leur) faire des histoires» (p. 48).

Cherchant désespérément à «improviser sur-le-champ un scénario passe-muraille» et à mettre du style dans ses feintes (p. 59), le hêros finit par répondre tant bien que mal aux questions de son interlocuteur: «Je parle, mais qu'est-ce que je dis au juste? C'est illogique. Mon improvisation oblique dans le genre allusif» (p. 60). Et il déraille:

C'est pénible cette conversation dont je fais les frais: je meuble je dis n'im̌porte quoi, je déroulè' la bobine, j'enchaîne et je tisse mon suaire avec du fil à retoródrè. Là viäimént j'exagère en lui racontant que je fais une dépression nerveuse et en me composant une physionomie de défoncé. Et toute cette histoire de difficultés financières, cette allusion à dormir debout à mes deux enfants et à ma femme que j'aurais abandonnés, décidément je lui raconte des sornettes... (p. 61):

Sornettes ou non, H. de Heutzz à l'air de mordre: «Je joue le tout pour le tout: je continue dans l'invraisemblable.... (p. 61). C'est ainsi que, profitant de l'hésitation et du doute suscités par son récit', le hẻrós parvient à désarmer són adversaire et à rémpơrter une prémière victoire:

Chose curieuse, sa victoire ne lui remonte pas le moral: «J'éprouve uṇe grande lassitude: un vague désir de suicide me revient. Jè sưis fatigué à la fin» (p. 67). Son problème consiste à faire avancer l'histoire: "«Une seùle chose me préoccupait alors, à savoir la méthode que je dêvais utilisè poưt tuer $H$, de Heut $z^{15}$ 》 ( $\mathrm{p}$. 67). Là encore, il s'agit bel et bien d'un problème narratif: le-narrateur ne saurait raconter la victoire de son double, de son délégué de pouvoir. Au contraire, il se met à penser à la «route qui va de Papineauville jusqu'à La Nations (p. 78) et à sa comparution prochaine au Palais de justice,-en Cour du Banc de la Reine: «Devant le juge, je devrai répondre et me disculper de l'obscuration suicidaire de tout un peuple; 
répondre de mes frères qui se sont donné la mort après la défaite de SaintEustache et de ceux qui n'en finissent plus de les imiter, tandis qu'un écran de mélancolie les empêche de voir le soleil qui éclaire La Nation en ce moment même» (p. 79). S'identifiant aux Patriotes, son récit, comme leur rébellion, «ressemble à l'entreprise poétique d'un homme devenu indifférent quant aux modalités de son échec 16 ». Il lui presse «d'en finir avec H. de Heutz et toute cette histoire» (p. 75), tout comme les Patriotes «ont voulu en finir avec l'humiliation qui nous accable encore aujourd'hui 17\%. Cependant, mauvais terroriste, cet assassin se comporte en parfait gentleman - «Vous pouvez faire vos prières, à condition que ce soit bref»" (p. 81) -, suivant une fois de plus l'exemple des «bons colonisés» de 1837: «Pas de coups bas, pas de 'furia francese'; pas de ruses ou si peu, pas de manières déplacées à table. On mange comme son hôte ${ }^{18}$ ».

En effet, c'est l'ennemi qui, dans ce deuxième affrontement, se sert de la ruse. En se transformant, dans le bois de Coppet, en François-Marc de Saugy, fondé de pouvoir et double du personnage créé par le héros, $\mathrm{H}$. de Heutz réussit à s'emparer de la narration de l'histoire. Son plagiat imprévu inspire au héros une sorte de vertige qui le paralyse et le désarme; c'est d'ailleurs un rôle que l'historien joue à la perfection: «En toute sincérité, je reconnais que $\mathrm{H}$. de Heutz fait preuve d'un art consommé. Cet homme possède un don diabolique pour falsifier la vraisemblance; si je n'étais pas sur mes gardes, il m'aurait à coup sûr et pourrait me convaincre qu'il est mon frère, que nous étions nés pour nous rencontrer et pour nous comprendre. J'ai vraiment affaire au diable» (p. 84-85). Diable ou frère, l'historien finit par fasciner, par séduire le héros. Moyennant un renversement insidieux du procédé schizophrénique, les deux hommes, ennemis' implacables, se fondent en un seul: identité anti-dialectique et déréalisante.

Chose intéressante: la ruse de l'adversaire est un tour de force essentiellement poétique; la part de la vraisemblance, de l'illusion référentielle, y est infime; c'est la forme même du récit, de cette «autobiographie incroyable», qui paralyse le héros en l'envoûtant 19 . Victime de la fatigue dialectique ${ }^{20}$, celui-ci s'affaisse devant le Mythos de l'Autre - cette narration au nom de quoi une idéologie politique «s'insurge contre le Logos - et contre sa dia-lectique $21_{\gg}$. Il tombe sous «l'étrange paralysie qui a frappé les vainqueurs de Saint-Denis, cette syncope soudaine qui a creusé un abîme d'hésitation entre la première bataille de 1837 et toutes les défaites qui lui ont succédé22》.

Encore une fois le caractère narratif de l'échec est souligné par un retour à la situation narrative et à l'impuissance du narrateur, qui constate que tout lui échappe, que rien n'est libre ici, pas même l'intrigue:

Quelque chose me dit qu'un modèle antérieur plonge mon improvisation dans une forme atavique et qu'une alluvion ancienne étreint le fleuve instantané qui m'échappe. Je n'écris pas, je suis écrit. Le geste futur me connaît depuis longtemps. Le roman incréé me dicte le mot à mot que je m'approprie, au fur et à 
mesure, selon la convention de Genève régissant la propriété littéraire: Je crée ce qui me devance et pose devant moi l'empreinte de mes pas imprévisibles. L'imaginaire est une cicatrice. (p. 89-90)

Le narrateur se rend ainsi compte que lui aussi, comme le personnage qu'il a créé, comme tout dominé, "vit un roman écrit d'avance23").

Après une fuite ignoble provoquée par «l'apparition magique d'une femme blonde» (p. 109) et suivie d'un repas copieux à l'Auberge des Émigrés, le héros se rend au château d'Echandens où aura lieu le troisième affrontement. Pour cette confrontation, il adopte en quelque sorte la stratégie de son ennemi, comptant sur la surprise et sur l'originalité de son plagiat: «Ma stratégie ne peut que les déconcerter: dans le genre, c'est un petit chef-d'oeuvre» (p. 115). La voiture de l'historien lui sert de cheval de Troie' pour s'introduire dans la «citadelle ennemie»: «Moi, agent révolutionnaire par deux fois pris au dépourvu, j'étais en quelque sorte déguisé en $H$. de Heutz, revêtu de sa cuirasse bleue, muni de ses fausses identités et porteur de ses clés héraldiques» (p. 115). Une fois de plus le caractère théâtral de son entreprise se voit mis en relief: «En fait, $\mathrm{j}$ 'avais le trac. Aviant d'entrer en scène, j'étais soudain la proie d'une agitation incon-' trôlable» (p. 116-117).

Une fois surmontée sa peur de comédien, l'agent révolutionnaire se trouve enfin en scène. Cependant, l'Histoire est, encore une fois, contre lui et son projet d' «invasion»: "L'erreur des Patriotes de 1838 ne se traduit pas par une carence de leur volonté de vaincre, ni par l'utilisation de tactiques désuètes; leur erreur a été de sous-estimer l'ennemi et de croire, implicite-' ment, que ce serait le même genre d'ennemi qu'en 183724.» Le Patriote a tout prévu', mais, pendant son attente, il se laisse de plus en plus envoûter par la puissance mystique de l'histoire et de la culture qui entourent son adversaire. Comme l'a dit Michel Bernard, les forces contrerévolutionnaires ont changé d'aspect au cours de la poursuite: «Au fur et à mesure que la lutte se développe, à travers les péripéties, le révolutionnaire bourgeois découvre sa ressemblance avec l'adversaire qu'il s'est donné, son ennemi complémentaire. Il mesure la fascination que continue d'exercer sur lui le monde de la culture et du confort de cette bourgeoisie. Il ne parvient pas réellement à combattre ce monde avec lequel il conserve mille attaches $25: »$ En effet, le réel échappe sans cesse à ce héros hanté par les mythes. Là encore, la dialectique risque d'avorter grâce à l'introduction d'un terme transcendant: «H. de Heutz ne m'a jamais paru aussi mystérieux qu'en ce moment même, dans ce château qu'il hante élégamment... l'homme qui demeure ici transcende avec éclat l'image que je me suis faite de' ma victime... Je suis aux prises avec un homme qui me dépasse» (p. 129).

Dans l'ex-libris indéchiffrable du livre du colonel Stoffel (l'Histoire de. Jules César, guerres civiles), dans «le caractère prémédité de ce chef-d'oeuvre de confusion» où il "dénombre une quantité incommensurable d'articulations» (p. 130-131), le héros se fait une nouvelle image de. 
l'homme qui habite' «ce château impossible» et qui détient la clé de l'énigme qu'est l'univers romanesque dans lequel ils se meuvent; «Il en est ainsi du château tout entier qui me mystifie non pas tellement en tant qu'habitacle, mais en tant que chiffre. Car ces coffres ciselés qui ne contiennent rien, ces médaillons qui réflètent des images de guerre et ce livre apparemment oublié qui raconte les combats de César, voilà autant d'initiales nouées inextricablement dans un fouillis hautain et fascinant. Tout cela porte une signature, celle de l'homme que j'attends» (p. 131). Sur ce «plan strictement littéraire», c'est $H$. de Heutz qui s'est emparé du code régissant le «système constellaire», l'alphabet dans lequel le narrateur a emprisonné son héros; c'est l'historien absent mais omniscient qui multiplie les inconnues de l'équation fictive.

La fatigue s'instaure de nouveau; le révolutionnaire finit par désaxer lui-même "la dialectique historique... en situant le pôle supérieur à un niveau très élevé26»; «cet homme m'échappe infiniment», se dit-il, paralysé par la «splendeur sarcophale» (p. 146) de la demeure dans laquelle il se trouve. Aux yeux de l' «adolescence sacrilège 27 », H. de Heutz revêt toute la puissance insaisissable du père omniscient: "Ce que je perçois de lui ne sera toujours qu'une infime portion de sa puissance. Ses épiphanies me déconcertent et me prennent invariablement au dépourvu. L'impression qu'il produit sur moi neutralise ma capacité de riposter. Pétri d'invraisemblance, $\mathrm{H}$. de Heutz se meut dans la sorcellerie et le mystère» ( $\mathrm{p}$. 134). « Empiégé dans un cachot festonné» (p. 137), le héros se rapproche de plus en plus de la situation du narrateur. Faute d'une cible vivante, d'un adversaire réel qui refuse la dialectique en s'absentant, il dirige sa colère dispersée contre l'image réifiée de $\mathrm{H}$. de Heutz: «J'ai envie de frapper au hasard, de trouer le guerrier nu d'une balle de revolver et de vider le restant du barillet dans le corps inférieur du buffet Louis XIII» (p. 136). Comme le narrateur avant lui, il invoque l'intensité de la violence pour remplir son propre néant; mais, sans objet réel, sans un ennemi à abattre, la violence s'éparpille, se tourne contre elle-même pour se consumer: «La violence m'a brisé avant que j'aie le temps de la répandre. Je n'ai plus d'énergie; ma propre désolation m'écrase. J'agonise sans style, comme mes frères anciens de Saint-Eustache... je suis sur le point de céder à la fatigue historique... J'ai besoin de H. de Heutz. S'il n'arrive pas, que vais-je devenir? Quand il n'est pas devant moi, en personne, j'oublie que je veux le tuer et je ne ressens plus la nécessité aveuglante de notre entreprise" (p. 139-40). L'échec, ici encore, est avant tout narratif: «Les coordonnées de l'intrigue se sont emmêlées. J'ai perdu le fil de mon histoire, et me voici rendu au milieu d'un chapitre que je ne sais plus comment finir» (p. 142).

Toute dialectique désaxée, tout affrontement noyé, il ne reste plus au héros qu'à jouer le rôle qui lui est dicté. Lá conversation téléphonique, avec ses dédoublements et l'identification sous-entendue de $\mathrm{K}$. avec la femme aux cheveux blonds, pousse à son point ultime la sensation de vertige du narrateur. qui a perdu la direction de son histoire. $\mathrm{H}$. de Heutz triomphe dans ce 'combat ritualisé et déréalisant en vertu d'une puissance supérieure 
et mystique qui lui est attribuée par son adversaire. Celui-ci reste, à la limite, enchaîné dans l'alphabet indéchiffrable du narrateur, pris dans une histoire dont il ne sera jamais l'auteur. Son retour à Montréal, son arrestation, son assimilation totale au narrateur interné, ne sont que les conséquences inévitables de l'Histoire, du Mythos, qui a fini par s'imposer.

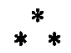

L'histoire de Prochain Épisode relève donc d'un art de la défaite: art stylistique, narratif, thêâtral, qui se confond à chaque instant avec une certaine conception de l'Histoire comme Mythos. Point n'est besoin d'insister ici sur les sources bien connues de l'analyse faite par Aquin de la situation coloniale. Que le romancier était un lecteur avide de Memmi, de Berque, de Senghor, de Mannoni, de Sartre, on le sait depuis longtemps. Cependant, il ne serait peut-être pas inutile de signaler ici un écho textuel de la voix de ce dernier dans Prochain Épisode. Les passages en question, qui se trouvent dans le Diable et le Bon Dieu, sont d'un intérêt particulier parce que l'on y trouve la même théâtralité déréalisante, la même identification invraisemblable et diabolique, la même transformation parodique de l'intersubjectivité en intertextualité, que nous avons constatées dans les combats ritualisés de Prochain Épisode. Il s'agit de deux échanges entre Goetz - cet «agent double de naissance» - et Heinrich, ce traître qui ne s'accepte pas, qui «joue double jeu», qui «pense en deux langues». Dans le premier de ces deux échanges, Goetz s'efforce de persuader Heinrich de lui dire comment on entre dans la ville de Worms:

\section{HEINRICH}

Tu n'existes pas. Tes paroles sont mortes avant d'entrer dans mes oreilles, ton visage n'est pas de ceux qu'on rencontre en plein jour. Je sais tout ce que tu diras, je prévois tous tes gestes. Tu es ma créature, et je te souffle tes pensées. Je rêve, tout est mort et l'air a goût de sommeil.

\section{GOETZ}

En ce cas, je rêve aussi car je te prévois si minutieusement que tu m'ennuies déjà. Reste à savoir lequel des deux habite le rêve de l'autre28.

La même métaphore du souffleur diabolique revient vers la fin de la pièce avec une résonance aquinienne toute particulière:

\section{HEINRICH}

Eh bien... (Un temps.) Attends: c'est un trou de mémoire. Je suis sujet à ces absences; ça va me revenir. (Il marche avec agitation.) J'avais pourtant bien pris mes précautions; ce matin j'ai tout repassé dans ma.tête... C'est ta faute: tu n'es pas comme tu devrais être. Il fallait que tu sois couronné de 
roses avec des yeux triomphants, j'aurais bousculé ta couronne et saccagé ton triomphe; à la fin, tu serais tombé sur les genous... Où est ta superbe? Où est ton insolence? Tu es à demi mort, quel plaisir veux-tu que je prenne à t'achever? (Avec rage.) Ah! Je ne suis pas encore assez méchant!

\section{GOETZ}

Tu te crispes, Heinrich, détends-toi, prends ton temps.

\section{HEINRICH}

Il n'y a pas une minute à perdre. Je te dis qu'ils sont sur mes talons. (Au Diable.) Souffle-moi, souffle-moi: aide-moi à le haïr de près. (Plaintivement.) Il n'est jamais là quand on a besoin de lui.

\section{GOETZ}

Moi, je vais te souffler ${ }^{29}$.

Une analyse des parallélismes s'établissant entre ces échanges et ceux de Prochain Épisode serait sans doute superflue ici. Il suffit de signaler l'influence probable de Sartre sur Aquin, influence sur laquelle les critiques n'ont peut-être pas assez insisté.

Nous avons constaté dans Prochain Épisode une conception de l'histoire du Canada français comme récit théâtral, régi en fonction d'une psychopathologie de l'échec, d'un Mythos de la défaite qui ne fait que répéter à l'mfini la scène primitive et déterminante des plaines d'Abraham. C'est ainsi que, dans une situation minoritaire caractérisée par la fatigue historique des dépossédés, le nationalisme deviendrait, selon Aquin, «un psycho-drame cathartique... une sorte d'irruption peccamineuse insérée à l'avance dans le système qu'elle conteste avec incohérence mais n'ébranle jamais ${ }^{30}$ ». Le Canada français, «encadré par une structure qu'il n'invente pas», «détiendrait un rôle, le premier à l'occasion, dans une histoire dont il ne serait jamais l'auteur», «une histoire faite par d'autres 31 », car «le dominé vit un roman écrit d'avance ${ }^{32}$ 》). C'est ce désaxement de la dialectique historique qui se voit reflété dans le récit d'espionnage de Prochain Épisode. De là on pourrait conclure, comme l'ont fait d'ailleurs la plupart des critiques, que l'échec de l'illusion référentielle de ce récit signifie l'échec de toute donnée révolutionnaire. Pourtant, cette interprétation, si cohérente soit-elle, ne tient pas compte d'un fait très important et hautement problématique, à savoir: le statut explicitement fictif de ce référent qui renonce à se réaliser.

Nous avons vu que le procès de récupération et d'assimilation impliqué par le Mythos de la défaite s'oppose au projet d'assassinat par l'inter- 
médiaire qu'on pourrait appeler la schizophrénie narrative du roman. Cette schizophrénie correspond, d'une part, à la dialectique du discours et de l'histoire (c'cst-à-dire des deux niveaux du texte) et, d'autre part, au conflit intérieur du narrateur, selon le théorème suivant: le coefficient d'échec (ou de non-génération) du récit d'espionnage serait directement proportionnel au coefficient de névrose (ou d'incohérence ontologique) du narrateur. Or, pour que cet échec ne soit qu'un reflet non problématique du mécanisme de récupération historique, il faudrait que la schizophrénie narrative soit dépourvue de toute possibilité d'autocritique. Mais nous avons vu que la bataille de la narration représente non seulement une constatation passive de la part du narrateur, mais encore une prise de conscience progressive du Mythos fondateur: prise de conscience qui prend la forme d'une critique, tantôt ironique, tantôt désespérée, du projet de roman. C'est en ce sens restreint que l'on peut parler d'une relation dynamique entre le narrateur interné et le héros du récit d'espionnage, relation qui pourrait être projetée sur les axes du discours et de l'histoire comme une spirale ascendante

Le projet de roman, nous l'avons dit, est explicitement fictif: il incarne non seulement la fable (dans le sens d'Éco: «une machine produisant de la redondance», fournissant «l'évasion en contant non ce qui est ignoré, mais du déjà connu ${ }^{33}$ )), avec toutes ses implications idéologiques (surtout l'incapacité de "passer de la mythologie à la raison34», du Mythos au Logos), mais aussi le passé à demi mythique du narrateur. Son échec et, partant, celui de la donnée révolutionnaire qu'il contient, ne peut donc être interprété que comme l'échec d'un projet d'évasion et comme un exorcisme des tentations compensatrices symbolisées dans le roman par le personnage de Ferragus.

La structure de Prochain Épisode se caractérise par une tension extrême à l'intérieur même du signe narratif. Une histoire qui se voudrait l'instrument d'une logo-thérapie compensatrice s'y trouve contestée par un discours qui hésite sans cesse à l'organiser. Par conséquent, l'univers fictif ne se construit pas selon le modèle projeté. Quelles sont les modalités de cet échec? L'histoire voudrait vénger une impuissance réellement vécue; le discours refuse cette vengeance littéraire en affirmant la réalité irréfutable de l'impuissance. L'histoire voudrait établir une dialectique avec l'ennemi; le discours démontre son impossibilité en invoquant le Mythos de lí. défaite par lequel l'ennemi, investi d'une supériorité accablante, peut refuser la dialectique. L'histoire aspire à la clôture de l'Oeuvre, à la cicatrice littéraire et anhistorique; le discours y oppose l'ouverture de la blessure quotidienne.

Ce discours qui hésite sans cesse à organiser une histoire insérée à l'avance dans le système qu'elle voudrait contester, c'est l'individu qui se trouve aux prises avec une structure qu'il n'invente pas et par laquelle il se trouve encadré; la crise narrative est une crise ontologique. Mais c'est aussi une crise historique, en ce sens que l'ontologie assumée par le narrateur de 
Prochain Épisode n'est rien d'autre que l'ontologie de son peuple. Bref, en ramenant sans cesse la fausse transcendance de l'histoire dans le cercle prédit d'une immanence intolérable, le discours constitue une prise de concience des structures mythiques d'un inconscient à la fois individuel et collectif: «Le 'système' qui nous enveloppe est subtil et diffus... À vrai dire, le 'système' repose d'abord sur les convictions inavouables et non-écrites de notre mentalité. Et ce sont ces fondations secrètes de l'ordre que nous visons. Nous sommes en présence d'un inconscient collectif, objet multiple de deux siècles de refoulement, qu'il nous presse de faire affleurer à la conscience ${ }^{35}$. Ainsi, comme l'a dit Michel Bernard, Prochain Épisode «se poset-il comme roman de la lucidité autocritique ${ }^{36}$ ).

La part de l'illusion littérale dans cette autocritique est, semble-t-il, paradoxale. Loin d'être une fin en elle-même - «le Graal esthétique qui fausse toute expédition» (p. 92) - elle n'est ici qu'un moyen, une manifestation critique de la souffrance littéraire comme «mode supérieur de connaissance ${ }^{37}$ ». L'illusion littérale ne conteste plus, comme le voudrait Ricardou, la vision humaniste 38 ; au contraire, elle l'approfondit en l'opposant à la réification de la machine normative des règles du genre. Ce qui ressort de cette dialectique, c'est une illusion référentielle décomposée qui correspond à la dévastation intérieure et à l'incohérence ontologique du narrateur et du peuple dont il est le «symbole fracturé» (p. 25).

Cette identité de structure entre le texte éclaté et une ontologie désolée se manifeste surtout dans le «style» de Prochain Épisode: «du fait de son enracinement, l'écrivain devra vraisemblablement être manifestement québécois, créer son mode de manifestation personnel, inventer le style de sa propre épiphanie... afin d'être (dans ses livres) québécois à rendre malade ${ }^{39}$ ». Mais cette valorisation du style, qui caractérise presque tous les écrits d'Aquin indépendamment du sujet traité, ne relève point d'un esthétisme aveugle aux réalités du monde extérieur. Il ne s'agit pas du style au sens habituel et restreint du terme; plus qu'un phénomène littéraire, le style est, pour Aquin, un phénomène ontologique, une façon d'être: «Le problème n'est pas d'écrire des histoires qui se passent au Canada, mais d'assumer pleinement et douloureusement toute la difficulté de son identité. Le Canada français, comme Fontenelle sur son lit de mort, ressent une certaine difficulté d'être 40 .» Assumer cette identité, vivre cette "difficulté d'être», voilà le sens profond de l'engagement d'Aquin, de son «appartenance», de son enracinement.

C'est cet engagement au réel qui s'oppose, à tous les niveaux, à la fatique dialectique, au Mythos de la défaite. Au niveau de l'écriture, c'est le quotidien qui s'affirme contre les règles du genre: «Ecrire un roman d'espionnage comme on en lit, ce n'est pas loyal: c'est d'ailleurs impossible. Écrire une histoire n'est rien, si cela ne devient pas la ponctuation quotidienne et détaillée de mon immobilité interminable et de ma chute ralentie dans cette fosse liquide.» (p.9) C'est le contexte politique qui s'oppose au prétexte et au précepte rhétoriques, au dogmatisme des schémas; c'est l'in- 
cohérence ontologique qui refuse l'algèbre et le mécanique. C'est l'individu qui se charge d'une intolérable crise d'identité collective:

En moi, déprimé explosif, toute une nation s'aplatit historiquement et raconte son enfance perdue, par bouffées de mots bégayés et de délires scripturaires et, sous le choc noir de la lucidité, se met soudain à pleurer devant l'immensité du désastre et l'envergure quasi sublime de son échec. Arrive un moment, après deux siècles de conquêtes et 34 ans de tristesse confusionnelle, où l'on n'a plus la force d'aller au-delà de l'abominable vision. Encastré dans les murs de l'Institut et muni d'un dossier de terroriste à phases maniaco-spectrales, je cède au vertige d'écrire mes mémoires et $\mathrm{j}$ 'entreprends de dresser un procès-verbal précis et minutieux d'un suicide qui n'en finit plus. (p. 25-26)

C'est le désespoir d'une impuissance qui veut être vécue, qui s'affirme contre toute tentative d'évasion littéraire, contre toute compensation esthétique. C'est le discours qui conteste une histoire déréalisante et déterminée à l'avance; c'est la réalité irréfutable de la situation narrative, d'un narrateur condamné à habiter l'antichambre d'une révolution ratée.

Le style de Prochain Épisode, à la fois exorcisme et baptême, décomposition et ouverture, est ce qui permet la transition de la "cohérence invisible» de l'histoire conçue comme Mythos anti-dialectique à l'avenir incertain et anarchique de la révolution: «Mon livre n'est pas le livre d'un déprimé politique au sens maladif du terme; et il n'est pas suicidaire puisque le prochain épisode, c'est la révolution à faire ${ }^{41}$.» Malheureusement, l'analyse textuelle ne saurait guère justifier cet optimisme. Comment se fier en toute bonne foi à ce narrateur vacillant, à ce «tabernacle impur» de la révolution? Mais, de même que la prise de conscience de la situation historique ne garantit nullement le passage à l'action, de même on ne saurait souscrire à cette hérésie de la «psychiatrie viennoise» que nous propose André Berthiaume: «Les ultimes envolées révolutionnaires ne trompent personne: le présent est garant de l'avenir ${ }^{42}$.» Évidemment, ce critique a choisi d'ignorer un aspect essentiel de la pensée d'Aquin, c'est-à-dire son existentialisme: «Au nom de tout un passé qu'on croit déterminant, on refuse un avenir que nous gardons pourtant le pouvoir de déterminer, du moins selon les principes de Saint-Thomas et de Jean-Paul Sartre ${ }^{43}$.» «Les peuples sont ontologiquement indéterminés, et cette indétermination est le fondement même de leur liberté. L'histoire à venir d'un groupe humain n'est pas fatale, elle est imprévisible. "Un homme se définit par son projet», a dit Jean-Paul Sartre. Un peuple aussi44.» En effet, on pourrait pousser plus loin cette comparaison:

JUPITER. - Que comptes-tu faire?

ORESTE. - Les hommes d'Argos sont mes hommes. Il faut que je leur ouvre les yeux.

JUPITER. - Pauvres gens! Tu vas leur faire cadeau de la solitude et de la honte, tu vas arracher les étoffes dont je les 
avais couverts, et tu leur montreras soudain leur existence, leur obscène et fade existence, qui leur est donnée pour rien. ORESTE. - Pourquoi leur refuserais-je le désespoir qui est en moi, puisque c'est leur lot?

JUPITER. - Qu 'en feront-ils?

ORESTE. - Ce qu'ils voudront; ils sont libres, et la vie humaine commence de l'autre côté du désespoir 45 .

En démythifiant les structures mentales de la collectivité canadiennefrançaise, en passant, par le truchement de la dialectique «génératrice de lucidité et de logique ${ }^{46}$ », à une prise de conscience de la situation minoritaire et de ses corollaires, Prochain Épisode a vécu ce désespoir. Cette prise de conscience, ce désespoir écrit, c'est tout ce dont le livre se croit capable. Le reste, le «désespoir agi» (p. 94), le «projet d'amour total», appartient à l'Histoire, à la narration collective, à quelque «récit hunique» qui changerait la face des nations.

1. Jean-Pierre Faye, Théorie du récit, Paris, Hermann, 1972, p. 15.

2. Ibid., p. 19-20.

3. Observations sur l'histoire de France, Nouvelle Édition, 1788, Tome premier. Cité par Faye, p. 18.

4. Faye, p. 19.

5. Jean-Pierre Faye, «Sartre et les Huns», les Lettres françaises, no. 1122 (du 10 au 16 mars 1966), p. 5, 7.

6. Hubert Aquin, «L'art de la défaite», Liberté, 7, janvier-avril 1965, p. 33-41.

7. Ibid., p. 33.

8. Ibid., p. 35 .

9. Ibid.

10. Ibid., p. 35-36.

11. Ibid., p. 37-39.

12. Hubert Aquin, «La fatigue culturelle du Canada français », Liberté, 4, no. 23, maj 1962, p. 317.

13. Hubert Aquin, Prochain Épisode, Montréal, Le Cercle du Livre de France, 1965. Nos références à cette édition seront données dans le texte de notre article.

14. La conférence donnée par $\mathrm{H}$. de Heutz à Genève porte sur «César et les Helvètes» et n'est pas sans intérêt pour le lecteur de Faye: «Peu avant l'équinoxe du printemps de 58, les Helvètes s'étaient réunis au nord du lac Léman, en vue d'un exode massif dans l'ouest de la Gaule chevelue. Cette concentration opérée à quelques milles de Genaba (Genève) avec l'intention de traverser le Rhône sur le pont de cette ville et d'enfreindre ainsi l'intégrité de la Gaule transalpine, détermina la conduite de César» (p. 12).

15. Ces mots font écho à ceux du narrateur au début du roman (p. 7): «Au fond, un seul problème me préoccupe vraiment, c'est le suivant: de quelle façon dois-je m'y prendre pour écrire un roman d'espionnage?» Inutile d'insister sur le sens de ce parallélisme.

16. "L'art de la défaite», p. 37-38.

17. Ibid., p. 38.

18. Ibid., p. 36.

19. Marta Dvorak a déjà signalé le caractère narratif de ce combat: «Ainsi le lecteur se rend compte que le combat des adversaires est un combat de récits. Tout ce qui est contesté, c'est la compétence narrative». Marta Dvorak, «Une analyse structurale», dans Québec littéraire 2, Hubert Aquin, Montréal, Guérin, 1976, p. 28. 
20. Nous renvoyons à «La fatigue culturelle du Canada français». Rappelons brièvement les traits les plus importants de cette fatigue dialectique et culturelle. Il s'agit essentiellement d'une dialectique qui se voit subvertie par le fait que le terme conservateur se situe à un niveau tellement supérieur à celui du terme contestateur 'qu'il revêt les apparences d'une puissance transcendante, voire mystique. Par rapport à cette «grandeur infinie», les forces antithétiques se trouvent non seulement paralysées, mais elles se sentent, par surcroît, coupables: elles se persuadent que leur révolte est le signe d'un état de péché, L'antithèse révolutionnaire se voit ainsi non seulement récupérée, mais récupérée avant le fait: lá situation dialectique reste non réalisée. On reconnaîtra chez le héros de Prochain Épisode les corollaires psychologiques d'une telle situation: «l'auto-punition, le masochisme, l'auto-dévaluation, la "dépression», le manque d'enthousiasme et de vigueur, autant de sous-attitudes dépossédées que des anthropologues ont déjà baptisées de fatigue culturelle» (p. 314).

21. Jean-Pierre Faye, Théorie du récit, p. 26.

22. «L'art de la défaite», p. 39.

23. Hubert Aquin, Point de fuite, Montréal, le Cercle du livre de France, 1971, p. 52. La citation est tirée de l'article «Profession: écrivain» paru dans Parti pris, vol. I, no. 4, janvier 1964, p. 23-31.

24. «L'art de la défaite», p. 40.

25. Michel Bernard, «Prochain Épisode» ou l'auto-critique d'une impuissance», Parti pris, vol. IV, no. 3-4, novembre-décembre 1966, p. 85.

26. «La fatigue culturelle du Canada français», p. $\mathbf{3 2 2 .}$

27 Ibid., p. 315.

28. J.-P. Sartre; le Diable et le Bon Dieu, ,Paris, Gallimard, 1951. (Acte I, deuxième tableau, scène IV.)

29. Ibid., Acte III, dixième tableau, scène IV.

30. «La fatigue culturelle du Canada français», p. 315.

31. Ibid., p. 317.

32. Point de fuite, p. 52.

33. Umberto Éco, «James Bond: une combinatoire narrative», Communications, 8, 1966, p. 90.

34. Ibid., p. 92.

35. Hubert Aquin, “Comprendre dangereusement», Liberté, 3, no. 17, novembre 1961, p. 679.

36. «Prochain Épisode ou 1'auto-critique d'une impuissance», p. 86.

37. «Le bonheur d'expression», Liberté, 3, no. 18, décembre 1961, p. 742.

38. Jean Ricardou, Pour uné théorie du nouveau roman, Paris, Éditions du Seuil, 1971, p. 64-65.

39. Hubert Aquin, «La mort de 1'écrivain maudit», Liberté, 11, mai-juin-juillet 1969, p. 29-30.

40. "La fatigue culturelle du Canada français», p. 320.

41. Point de fuite, p. 16-17.

42. Añdré Berthiaume, «Le thème de l'hésitation dans Prochain Épisode», Liberté, vol. 15, no. 85 , février 1973 , p. 148.

43. Hubert Aquin, «L'existence politique», Liberté, 4, no. 21, mars 1962, p. 67.

44. "La fatigue culturelle du Canada français», p. 308.

45. Jean-Paul Sartre, les Mouches, Acte III, scène II, (Théatre, Paris, Gallimard, 1962, p. 72:)

46. «La fatigue culturelle du Canada français», p. 300. 